\title{
Enabling ICME: Successful Transition of Advanced Technologies to Engineering Practice
}

\author{
Mei Li
}

Integrated Computational Materials Science \& Engineering (ICME) has emerged as an important discipline in recent years due to its great potential in efficiently engineering new products and manufacturing processes. ICME methodology could identify the gaps in the integration process when applied to solve fundamental engineering problems. This will potentially lead to the advancement in fundamental understanding of the problems, development of the linkage along the processing chain, development of computational tools for engineering practice, and establishment of cyberinfrastructure for global information sharing. However, enabling ICME is also a great challenge which requires collaborative efforts within the global materials and engineering community.

In 2006, JOM published a series of articles to introduce ICME as a new approach and demonstrate the effectiveness of ICME in solving complex engineering problems through a couple of selected case studies. Since then, the global awareness of the methodology and activities around ICME have grown. The ICME topic of this issue will present progress made in recent years in the global efforts in developing ICME tools and essential elements, and in the successful transition of advanced technologies to engineering practice.

The first paper by John Allison provides a perspective on progress made and future activities toward the development of a more widespread ICME capability. As Allison describes in his article, substantial progress has been made in educating policy makers and the broader materials research community of the ICME approach to industrial problems. Important future steps to nurture this development include broadening the involvement to include the larger materials research community, incorporation of ICME into educational curricula, and development of an ICME cyberinfrastructure and commercial ICME software.

The growing global activities in developing ICME tools to solve complex industrial problems are demonstrated in the second paper by Baicheng Liu and collabrators from China and the third

\section{ICME technologies}

can be thought of as

being comprised of not

only purely technical

computational model

development, but also

the critical aspect of

supply-chain technology and dynamics.

paper by Dirk Helm and collaborators from Germany. Liu's paper describes the advances in multi-scale modeling of solidification and casting processes, as well as engineering applications to aluminum casting for the automotive industry, large steel ingot for the energy industry, and directionally solidified turbine blade casting for the aviation industry. The third paper describes the theory of developing microstructure- based materials models for metal deformations, the approach of linking the discrete dislocations, grain and phase boundaries to the continuum plasticity, as well as the applications along the processing chain of an automotive component manufactured from a dual phase steel.

With the growing involvement of the global materials science and engineering community, materials informatics becomes an important approach in storing and sharing information. The fourth paper by Surya R. Kalidindi and collaborators describes their work in microstructure informatics. It focuses on the development of novel algorithms and computationally efficient protocols that are capable of mining the essential information in large microstructure datasets and building robust knowledge systems that can be easily accessed, searched, and shared by the broader materials community.

The fifth paper by David Furrer and John Schirra provides perspectives in enabling successful implementation and sustainment of ICME in industrial applications and design systems. ICME technologies can be thought of as being comprised of not only purely technical computational model development, but also the critical aspect of supply-chain technology and dynamics. A complete and capable supply-chain is essential to the success of ICME.

Mei Li is Technical Expert and Group Leader of Light Metal Research at Ford Motor Company in Dearborn, Michigan and technical advisor for of the TMS Integrated Computational Materials Engineering Committee.

\section{Mei $\mathrm{Li}$ is a TMS Member!}

To read more about her, turn to page 7. To join TMS, visit www.tms.org/Society/Membership.aspx.

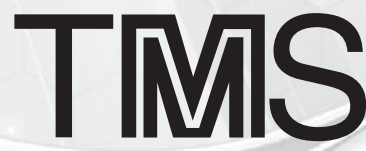

